\title{
Melioidosis fatalities in captive slender- tailed meerkats (Suricata suricatta): combining epidemiology, pathology and whole-genome sequencing supports variable mechanisms of transmission with one health implications
}

Audrey Rachlin ${ }^{1 *}$, Cathy Shilton², Jessica R. Webb ${ }^{1}$, Mark Mayo $^{1}$, Mirjam Kaestli ${ }^{1,3}$, Mariana Kleinecke', Vanessa Rigas', Suresh Benedict ${ }^{2}$, lan Gurry ${ }^{4}$ and Bart J. Currie ${ }^{1,5}$

\begin{abstract}
Background: Melioidosis is a tropical infectious disease which is being increasingly recognised throughout the globe. Infection occurs in humans and animals, typically through direct exposure to soil or water containing the environmental bacterium Burkholderia pseudomallei. Case clusters of melioidosis have been described in humans following severe weather events and in exotic animals imported into melioidosis endemic zones. Direct transmission of B. pseudomallei between animals and/or humans has been documented but is considered extremely rare. Between March 2015 and October 2016 eight fatal cases of melioidosis were reported in slender-tailed meerkats (Suricata suricatta) on display at a Wildlife Park in Northern Australia. To further investigate the melioidosis case cluster we sampled the meerkat enclosure and adjacent park areas and performed whole-genome sequencing (WGS) on all culture-positive B. pseudomallei environmental and clinical isolates.

Results: WGS confirmed that the fatalities were caused by two different B. pseudomallei sequence types (STs) but that seven of the meerkat isolates were highly similar on the whole-genome level. Used concurrently with detailed pathology data, our results demonstrate that the seven cases originated from a single original source, but routes of infection varied amongst meerkats belonging to the clonal outbreak cluster. Moreover, in some instances direct transmission may have transpired through wounds inflicted while fighting.

Conclusions: Collectively, this study supports the use of high-resolution WGS to enhance epidemiological investigations into transmission modalities and pathogenesis of melioidosis, especially in the instance of a possible clonal outbreak scenario in exotic zoological collections. Such findings from an animal outbreak have important One Health implications.
\end{abstract}

Keywords: Burkholderia pseudomallei, Melioidosis, Northern Australia, Whole-genome sequencing, Source tracing, Slender-tailed meerkats, Outbreak

\footnotetext{
* Correspondence: audreynicole.rachlin@menzies.edu.au

${ }^{1}$ Menzies School of Health Research, Charles Darwin University, Darwin,

Casuarina NT 0811, Australia

Full list of author information is available at the end of the article
}

(c) The Author(s). 2019 Open Access This article is distributed under the terms of the Creative Commons Attribution 4.0 International License (http://creativecommons.org/licenses/by/4.0/), which permits unrestricted use, distribution, and reproduction in any medium, provided you give appropriate credit to the original author(s) and the source, provide a link to the Creative Commons license, and indicate if changes were made. The Creative Commons Public Domain Dedication waiver (http://creativecommons.org/publicdomain/zero/1.0/) applies to the data made available in this article, unless otherwise stated. 


\section{Background}

Melioidosis is a disease of significant public health importance throughout much of the tropics, most notably in Southeast Asia and Northern Australia where it is considered highly endemic in both humans and animals [1]. The majority of cases arise through direct percutaneous exposure to the infections aetiological agent, Burkholderia pseudomallei [2], though case reports associated with severe weather events [3] and contaminated drinking supplies [4] have implicated inhalation and ingestion as potentially significant sources of infection. Recent studies from Thailand have raised the possibility that ingestion of water contaminated with $B$. pseudomallei may be a more common infecting event than previously thought, especially in endemic regions with unchlorinated water supplies [5]. Disease severity and clinical manifestations vary widely and in addition to route of infection, are influenced by host susceptibility, infecting bacterial dose and differential B. pseudomallei strain virulence [6, 7].

In humans melioidosis is being increasingly recognised as an opportunistic infection, with severe disease and death very uncommon in a healthy human host provided there is timely diagnosis and access to appropriate antibiotics [8]. In animals, the disease has now been documented in over 50 species $[9,10]$ with notable variations in disease susceptibility $[9,11,12]$. Animals native to melioidosis-endemic regions are generally resistant to disease despite frequent exposure to B. pseudomallei in the environment, while many exotic animals imported into zoos in endemic regions are especially susceptible and develop severe and often rapidly fatal disease [9, 11-14]. Melioidosis has been reported in a wide-array of livestock and in domestic pets $[9,10,12]$. Surveillance from tropical Northern Australia and Thailand has also shown that sheep [15], camels [16] and alpacas [17] readily succumb to melioidosis, while goats have diverse presentations similar to humans $[12,18]$ and pigs can often have asymptomatic internal infections [19].

Although zoonotic transmission of $B$. pseudomallei and direct transmission between animals is considered rare, outbreaks related to exposure of a contaminated environmental source have been reported in both endemic and non-endemic settings, including piggeries in Queensland, Australia [19], non-human primates in European and Asian zoos [13, 20], and previous incidents in captive slender-tailed meerkats on display in eastern Thailand [21]. Regular outbreaks observed in intensive livestock farms [19] and in zoos [14] suggest that stressful conditions may initiate the onset of disease, particularly in exotic non-native species.

High-resolution molecular fingerprinting tools have recently been used to investigate melioidosis case cluster and outbreak scenarios in both humans and animals [22-25]. Multi-locus sequence typing (MLST) is a popular, widely applicable genotyping tool with a global online database for assessing $B$. pseudomallei strain relatedness and melioidosis source attribution (http:// pubmlst.org/bpseudomallei) [26-28]. Despite this, there are instances where MLST is unable to determine finescale population structures due to exceedingly high rates of recombination in the B. pseudomallei genome [29, 30]. Phylogenetic reconstruction of B. pseudomallei populations using whole-genome sequencing (WGS) data can provide a highly robust method of outbreak source attribution and strain tracing on both an inter and intracontinental level [31-33]. Such technology is also highly applicable should a biothreat scenario arise involving this tier 1 select agent (http://www.selectagents.gov/). Additionally, WGS is now being used to investigate the genomic basis of differential virulence amongst $B$. pseudomallei isolates [7].

In this study, WGS and comparative genomic analysis were used to examine a cluster of eight fatal melioidosis cases reported in imported African slender-tailed meerkats (Suricata suricatta) on display at a Wildlife Park in Darwin, Northern Territory (NT), Australia. We undertook six rounds of environmental sampling in relation to the cases and performed MLST and WGS on all culturepositive B. pseudomallei environmental and clinical isolates. Used in conjunction with pathology data, we established the most likely modes of infection and transmission responsible for the outbreak.

\section{Results}

\section{Pathology and bacterial culture}

Significant pathological findings are detailed for each case in Table 1. Five of the meerkats had superficial erosive to deep ulcerative necrotising suppurative skin lesions with associated cellulitis, usually involving multiple sites, commonly including the distal legs in the region of the carpi or tarsi, the scrotum and the tail (Fig. 1a). In case \#3, \#4 and \#5, the skin lesions were associated with fibroplasia, and were therefore judged to be the oldest lesions present (at least 3 days duration). In two of these cases, B. pseudomallei was cultured from a skin lesion. In case $\# 8$, although there was a severe tarsal lesion from which B. pseudomallei was cultured, the lesion appeared relatively acute with no associated fibroplasia. In the fifth case with skin lesions (case \#6), the lesions were mild and considered likely incidental.

The liver exhibited lesions in all meerkats, with two distinct patterns. In four of the cases (cases \#1, $\# 2$, \#6 and \#8), extrahepatic and/or intrahepatic portal veins contained thrombi infiltrated with neutrophils, necrosis of the vessel wall and large suppurative foci centred on remains of portal triads (Fig. 2b). In cases $\# 6$ and $\# 8$ there was associated fibroplasia. In case \#2, in addition to the suppurative 
Table 1 Meerkat (Suricata suricatta) case details

\begin{tabular}{|c|c|c|c|c|c|}
\hline $\begin{array}{l}\text { Case } \\
\text { No. }\end{array}$ & $\begin{array}{l}\text { Date } \\
\text { of death }\end{array}$ & $\begin{array}{l}\text { Sex } \\
\text { Age }\end{array}$ & Pathology diagnoses & $\begin{array}{l}\text { B. pseudomallei positive } \\
\text { culture }\end{array}$ & $\begin{array}{l}\text { Suspected route } \\
\text { of exposure }\end{array}$ \\
\hline 1 & $\begin{array}{l}\text { Mar. 18, } \\
2015\end{array}$ & $\begin{array}{l}\text { Male, } 3 \\
\text { yrs. }\end{array}$ & $\begin{array}{l}\text { Liver: Multifocal intrahepatic portal suppurative thrombosis and } \\
\text { random multifocal suppurative hepatitis } \\
\text { Lung: Single large suppurative focus contiguous with } \\
\text { suppurative venous thrombus; random multifocal suppurative } \\
\text { pneumonia }\end{array}$ & $\begin{array}{l}\text { Bone marrow (femur), } \\
\text { spleen, lung }\end{array}$ & Ingestion (portal lesion) \\
\hline 2 & $\begin{array}{l}\text { April 23, } \\
2016\end{array}$ & $\begin{array}{l}\text { Female, } \\
10 \text { mos. }\end{array}$ & $\begin{array}{l}\text { Stomach: Transmural haemorrhagic, suppurative gastritis with } \\
\text { adjacent cranial mesenteric suppurative peritonitis with } \\
\text { fibroplasia } \\
\text { Liver: Multifocal intrahepatic portal suppurative thrombosis and } \\
\text { random multifocal suppurative hepatitis } \\
\text { Lung: Multifocal suppurative pneumonia, often associated with } \\
\text { haemorrhage and septic thrombosis }\end{array}$ & $\begin{array}{l}\text { Cranial mesentery, lung, } \\
\text { spleen }\end{array}$ & $\begin{array}{l}\text { Ingestion (gastric and } \\
\text { portal lesions) }\end{array}$ \\
\hline 3 & $\begin{array}{l}\text { April 26, } \\
2016\end{array}$ & $\begin{array}{l}\text { Male, } 10 \\
\text { mos. }\end{array}$ & $\begin{array}{l}\text { Skin: Multifocal severe, ulcerative, suppurative deep dermatitis } \\
\text { and cellulitis with associated fibroplasia (left forearm and left }\end{array}$ & $\begin{array}{l}\text { Left hind foot lesion, } \\
\text { lung, spleen }\end{array}$ & $\begin{array}{l}\text { Cutaneous (forearm and } \\
\text { hind foot lesions) }\end{array}$ \\
\hline
\end{tabular}
hind foot). Right scrotum open and draining necrohaemorrhagic exudate

Liver: Random multifocal suppurative hepatitis

Lung: Multifocal suppurative pneumonia, variably associated with haemorrhage and septic thrombosis

4 July 13, Female, Skin: Unhealed distal tail amputation with associated suppurative 201612 mos. deep dermatitis, fibrosis and bone remodelling; Focal ulcerative dermatitis (dorsal to left eye); Bilateral dorsal carpal epidermal hyperplasia and hyperkeratosis.

Left stifle: Suppurative arthritis

Liver and lung: Random multifocal suppurative hepatitis and pneumonia

Kidney: Segmental pyelonephritis

5 July 25, Male, 12 Skin: Multifocal subacute to chronic, ulcerative, suppurative 2016 mos. dermatitis (most severely involving the scrotum and perineum, more superficially involving the bridge of nose, above left eye, left ear pinna, tail, dorsal aspects both carpi and all feet, scrotum and perineum)

Liver and lung: Random multifocal suppurative hepatitis and pneumonia

6 Sept. 21, Male, 15 Liver: Multifocal portal suppurative thrombosis with associated 2016 mos. fibroplasia

Lung: Random multifocal suppurative pneumonia ung, spleen hind foot lesions)

Skin: Mild multifocal erosive dermatitis (ventral scrotum, media hind foot)

7 Oct. 7, Female, Intestine: Segmental transmural necrotising, suppurative enteritis 20163 yrs. with regional suppurative lymphadenitis

Lumbar vertebral column: Multifocal suppurative thrombosis and osteomyelitis with extension into vertebral canal Femur: Suppurative osteomyelitis extending to periosteum with associated fibrosis

Lung: Random multifocal suppurative pneumonia

8 Oct. 23, Female, Skin: Ulcerative, necrotising, suppurative deep dermatitis and 201616 mos. cellulitis (left tarsal region)

Vertebral column: Multifocal suppurative osteomyelitis

Lung: Random multifocal suppurative pneumonia

Liver: Extrahepatic portal vein suppurative phlebitis and thrombosis with associated fibroplasia. Liver otherwise too decomposed to assess

Lung, spleen positive. Perineal/scrotal swab negative

Liver, lung, spleen

Blood, liver, lung positive. Rectal and throat swabs negative

Left tarsal lesion swab, liver, lung, rectal swab, throat swab
Ingestion (portal lesion)

Ingestion (suppurative enteritis with regional suppurative lymphadenitis)

Cutaneous (multiple chronic skin lesions)

Ingestion (portal lesion) or cutaneous (tarsal lesion)

Signalment, history, pathological findings and B. pseudomallei culture results from the eight meerkat cases. ${ }^{1}$ Unless otherwise specified, the tissues noted as culture positive are all the tissues for which culture was attempted.

thrombi in portal veins, there was also severe transmural suppurative gastritis and contiguous cranial mesenteric suppurative peritonitis with associated fibroplasia (Figs. 1b, 2a). The second distinct pattern of liver lesion, which was present in all meerkats to a variable degree, including some with concurrent portal suppurative thrombosis, was acute random multifocal suppurative hepatitis (Figs. 1c, 2c). B. pseudomallei was cultured from every sample of liver for which culture was pursued (Table 1). 


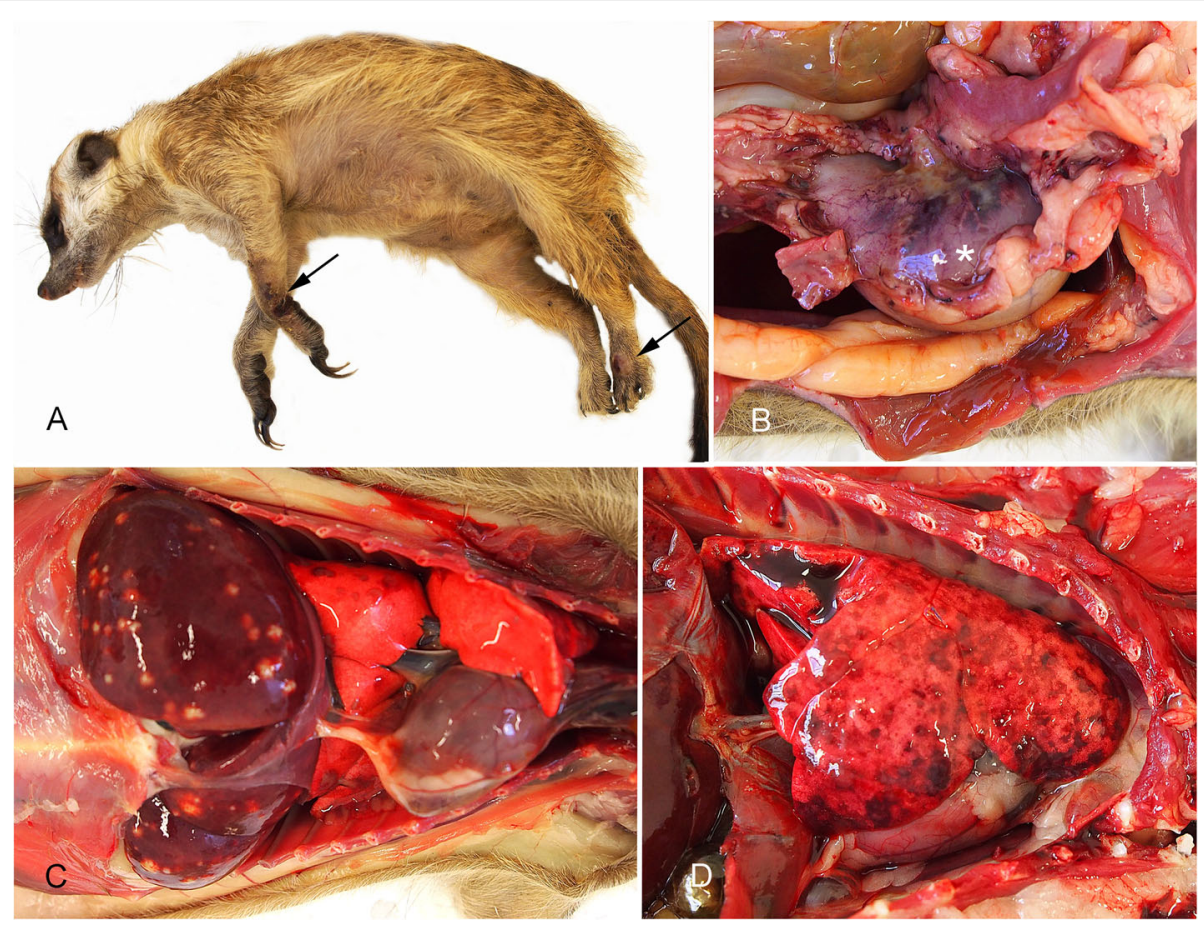

Fig. 1 Gross pathology of melioidosis in meerkats. Except for image A, all meerkats are positioned with the head to the right of the image. (a.) Typical ulcerative skin lesions involving left carpus and dorsal aspect of left hind foot (arrows) in case \#3. (b.) Stomach of case \#2 (asterisk) showing severe haemorrhage involving lesser curvature and purulent exudate in adjacent mesentery. (c.) Multifocal pale suppurative lesions throughout the liver in case \#4. Note also multiple small circular red foci of embolic infection in the lungs. (d.) Lung of case \#6 with abundant coalescing red foci, many with central pallor, representing severe, diffuse embolic showering of the lung

Lung lesions were present in all meerkats, and were generally similar, grossly appearing as $1-2 \mathrm{~mm}$ pale foci surrounded by red rims, distributed either near the free edges of lung lobes, or diffusely (Fig. 1d). Histologically, all lung lesions appeared as multifocal variably-sized random foci of neutrophil infiltration, frequently with surrounding haemorrhage and consistent with bacteraemic spread to the lungs (Fig. 2d). Some pulmonary lesions were associated with large thrombosed, necrotic vessels infiltrated with neutrophils. B. pseudomallei was cultured from lung in all eight cases. Although B. pseudomallei was cultured from every spleen sample in which culture was pursued (Table 1), the spleens were grossly normal, and histological findings limited to congestion and patchy increase in neutrophils within red pulp sinuses.

Histological examination of the vertebral column in two meerkats (cases \#7 and \#8) that clinically exhibited paresis or lameness involving the hind legs, revealed multifocal necrosis and neutrophil infiltration of the bone marrow, in one case with copious suppurative exudation expanding through the cortex into the spinal canal.

Specific bacterial culture aimed at detecting B. pseudomallei in samples from the pharyngeal, gastric, rectal, blood, and skin lesions samples from the two surviving meerkats was negative for all samples.

\section{Environmental sampling}

B. pseudomallei was isolated from three of the 64 environmental samples $(3 / 64,4.7 \%)$ collected at the park as part of the investigation. Two were from separate soil samples isolated from a garden bed in front of the meerkat enclosure and were collected approximately one month apart. The third was isolated from an air sample retrieved from a nature strip along the Wildlife Park parking lot during a low-pressure monsoon trough, less than $100 \mathrm{~m}$ from the meerkat enclosure (Fig. 3, Additional file 2: Data set S1).

\section{ST-36 is the strain responsible for infection in all but one meerkat}

Using the described in-house SNP ST PCR assays, $B$. pseudomallei isolates from seven of the eight (7/8, $87.5 \%)$ available meerkat isolates were designated as ST36 [31]. The isolate from the first meerkat infected at the park (MSHR8750) was classified as ST-562. ST-36 was also assigned to the air sample isolate taken from the Wildlife Park parking lot, while the two soil sample 


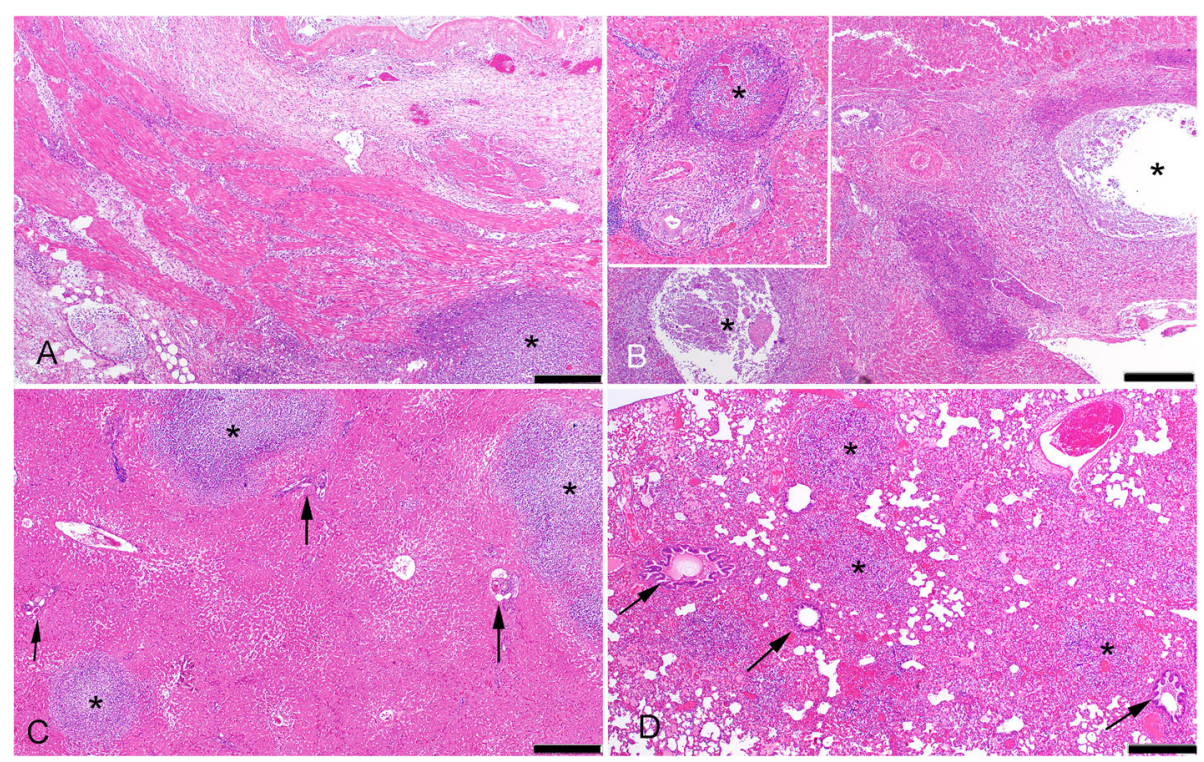

Fig. 2 Histopathology of melioidosis in meerkats. All main images are low power (40X) views, bars =500 $\mu \mathrm{m}$. (a.) Gastric wall of case \#2 (also depicted grossly in Fig. 1b). The lumen is at the top right of the image, serosa near the lower left. There is marked congestion and transmural oedema, with vascular thrombosis and foci of necrotic neutrophils in the wall (asterisk). The muscular tunics and serosa are mildly expanded by early fibroplasia. (b.) Entrance of portal vein (asterisks indicate two sections of the lumen) at the hepatic hilus in case \#6. The architecture of the vein is obliterated by a dense infiltrate of necrotic neutrophils expanding into the surrounding tissue, which is expanded by early fibroplasia. The inset shows a higher magnification of a portal triad with thrombosed intrahepatic tributary of the portal vein largely obliterated by an infiltrate of necrotic neutrophils (asterisk, note portal arteriole and bile duct to lower left of the vein). (c.) Liver from case \#4 (also depicted grossly in Fig. 1c). Suppurative foci (asterisks) in this case are random, typical of embolic showering of bacteria from the systemic circulation. Note unaffected nearby portal triads (arrows). (d.) Embolic showering of the lung in case \#6 (also depicted grossly in Fig. 1d) in which the primary lesion was likely via the portal circulation (depicted in Fig. 2b). Multiple random foci of neutrophil infiltration (asterisks) with surrounding haemorrhage. Note relatively unaffected nearby bronchioles (arrows)

isolates both originating from the garden bed across from the enclosure were designated as ST-132 (Fig. 4).

\section{WGS analysis reveals some clonality between ST-36 meerkat isolates}

To investigate whether the seven ST-36 meerkats were infected by a single point-source a comparative phylogeny was constructed using WGS data (Fig. 5). Six additional ST-36 human, animal and environmental isolates, two of which had previously been isolated from the same Darwin, Australia Wildlife Park, were included as close references. Maximum parsimony phylogenetic reconstruction of the ST-36 isolates identified 194 total orthologous SNPs and InDels across the 13 genomes. For the seven meerkat isolates, only 22 total orthologous SNP and InDel variants were identified and the isolates clustered closely together on one branch of the tree, indicating they were from the same lineage. Some chronological clustering between five of the isolates (MSHR9253, MSHR9256, MSHR9460, MSHR9647, MSHR9650) was also observed. Additionally, only a single variant was detected between two of the meerkats (MSHR9253; case \#2 and MSHR9256; case \#3), both of whom died of their infections three days apart in April 2016. Similarly, both fatal cases occurring in October 2016 (MSHR9647; case \#7 and MSHR9650; case \#8) were infected with closely related B. pseudomallei isolates, differing by only five variants. Although the blackcapped capuchin isolate (MSHR7590) and air isolate (MSHR10004) were both collected from the same Wildlife Park, they did not cluster with the meerkat isolates and varied by more than 40 orthologous SNPs and InDels from one another.

Lastly, we investigated the presence of the variably present fhaB3 gene and mutually exclusive $b i m A_{B p}$ and $\operatorname{bim} A_{B m}$ genotypes [34], virulence factors used as determinants of geographic origin and pathogenicity [7]. The seven meerkats with ST-36 B. pseudomallei infections were designated as carriers of the $\operatorname{bim} A_{B p}$ gene variant, as was the meerkat with the ST-562 infection (MSHR8750). All eight meerkat isolates including the ST-562 meerkat were also negative for the fhaB3 virulence gene. This is in contrast to most Australian $B$. pseudomallei isolates overall, but is seen commonly in ST-36 [7]. The four human, one capuchin and one air sampler ST-36 isolates were also negative for the fhaB3 gene variant. 


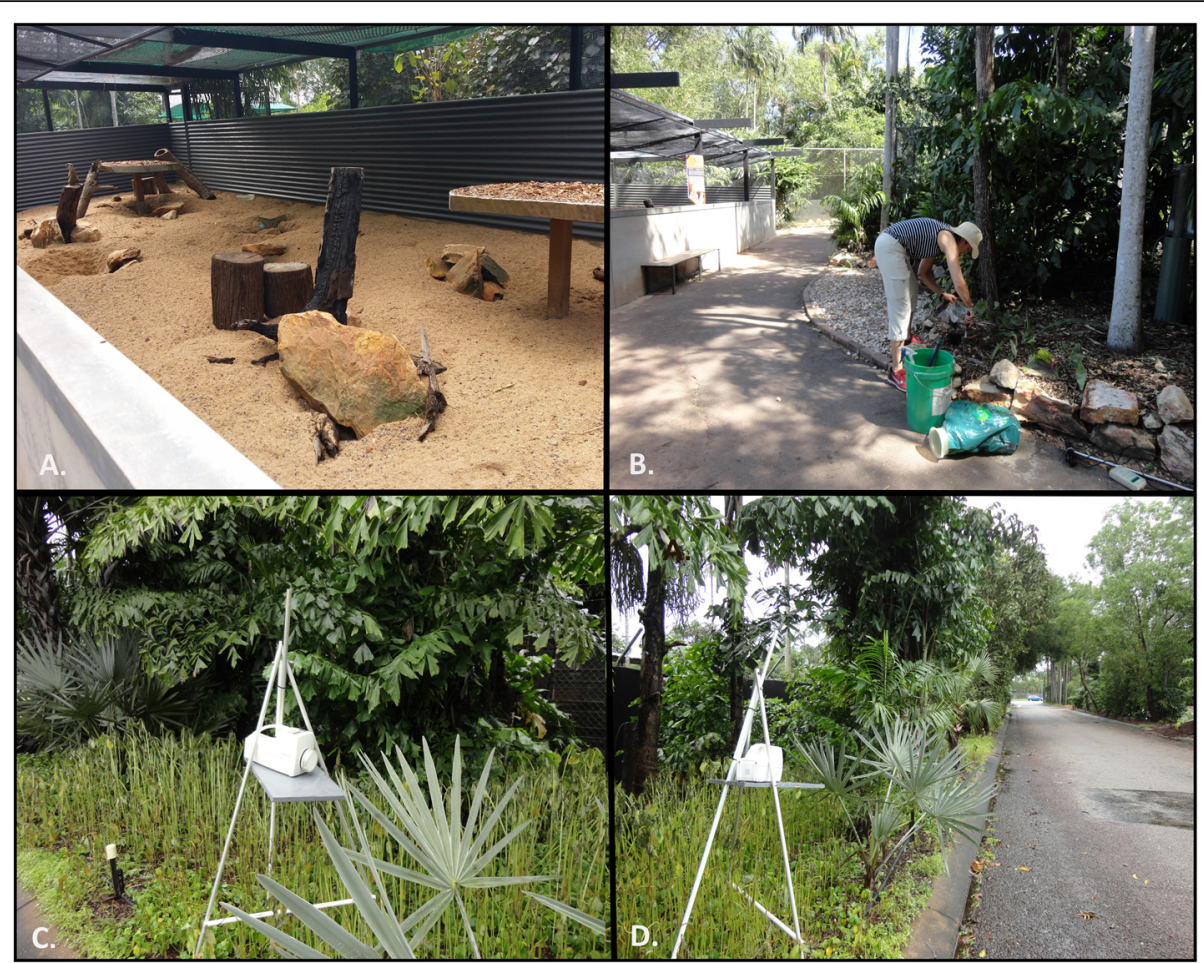

Fig. 3 Environmental sampling and isolation of B. pseudomallei at the Wildlife Park. Image of meerkat enclosure (a) and locations where B. pseudomallei was isolated in relation to the case cluster investigation. Image (b) displays the garden bed in front of the enclosure where the two positive soil samples were collected, while images (c) and (d) show the set-up and positioning of the positive air sample collected from the parking lot

\section{Discussion}

Unlike many other bacterial infections, melioidosis is not normally considered communicable, with only a small number of zoonotic, human-to-human and animal-to-animal cases reported [2, 35, 36]. Since the eight fatal meerkat B. pseudomallei infections occurred over a short time frame, this prompted suspicion of a single contaminated environmental source within the park enclosure. To fully investigate the aetiology of the case cluster, high-resolution WGS and detailed pathology data were combined to identify two separate environmental sources of $B$. pseudomallei infection with suggested variable routes of transmission. Only one of the B. pseudomallei strains, ST-36, was implicated in the large case cluster observed in seven of the meerkats. Despite this, extensive sampling efforts at the Wildlife Park failed to reveal the exact origin of the infections, with neither of the two B. pseudomallei ST-132 garden

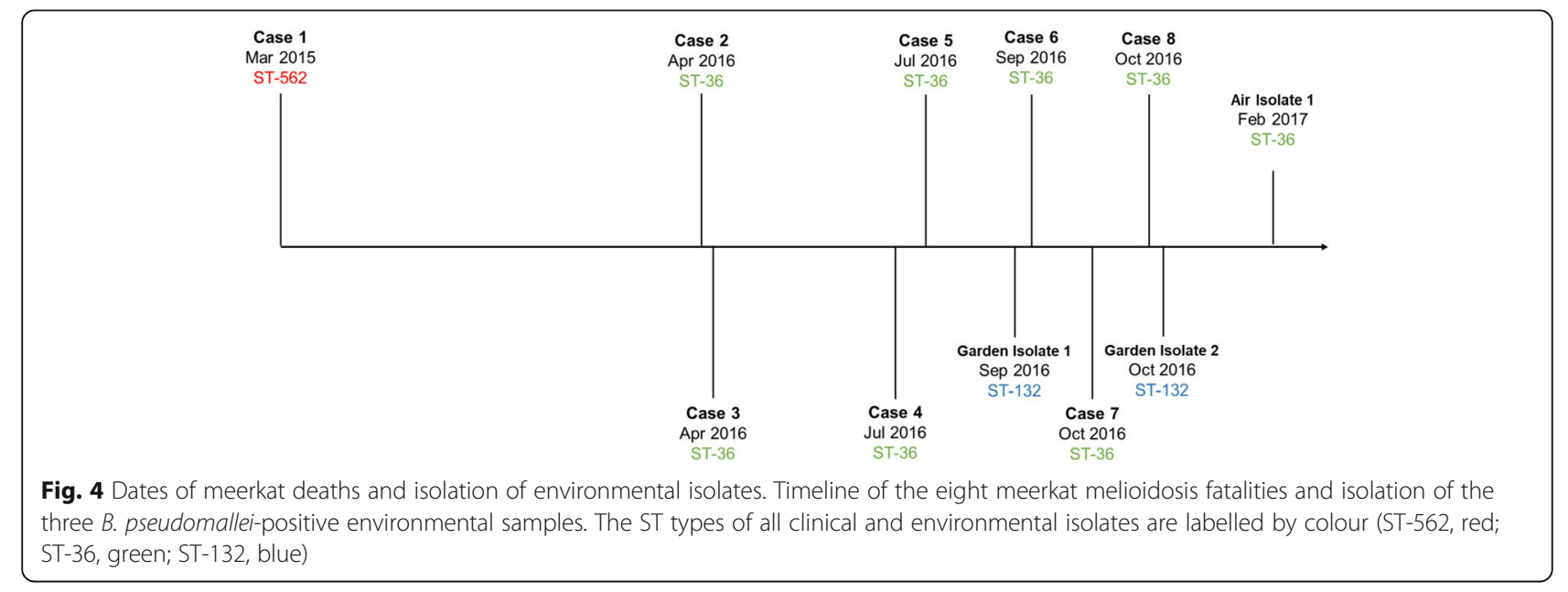


Tree scale: 10

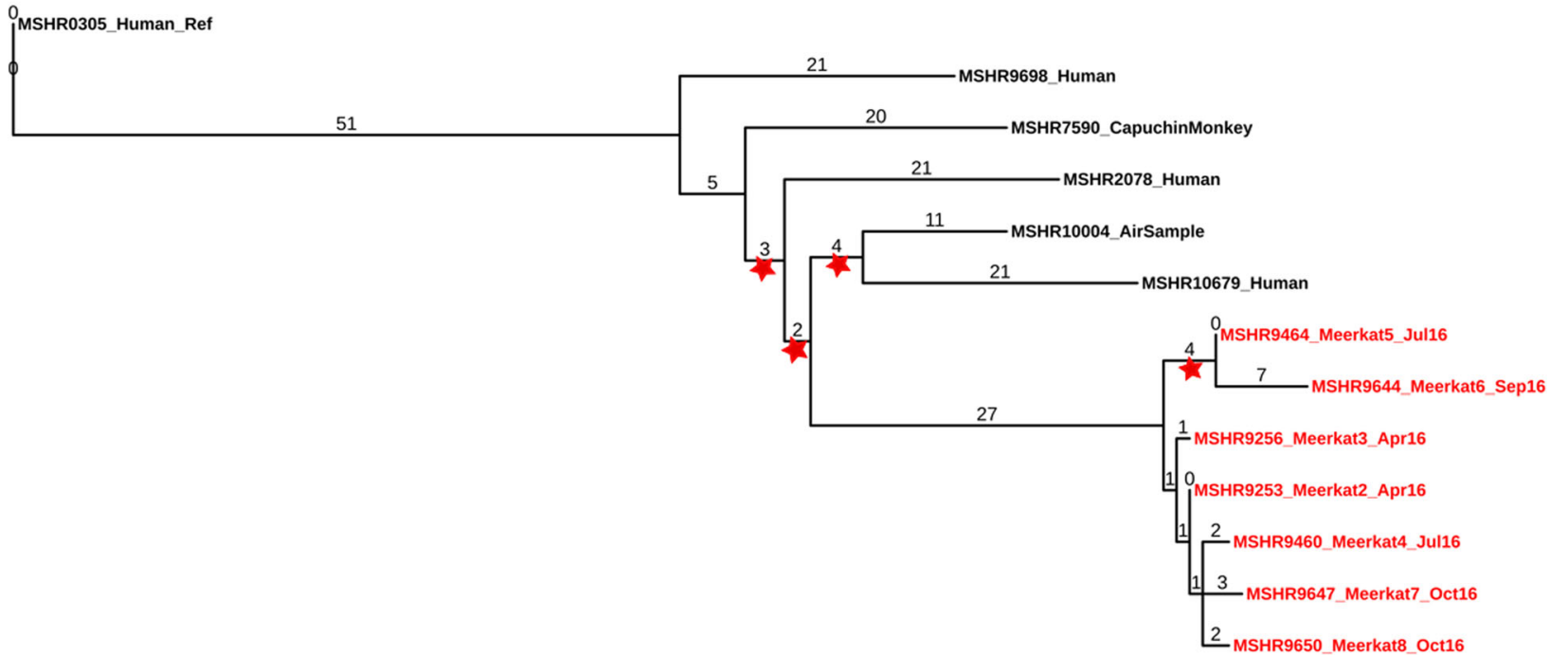

Fig. 5 Phylogenetic reconstruction of B. pseudomallei ST-36 genomes. Of the eight meerkats, seven with ST-36 B. pseudomallei genomes were included in the phylogeny. Maximum parsimony reconstruction of 13 B. pseudomallei ST-36 genomes was performed using 194 core-genome orthologous SNPs and InDels (overall consistency index $(\mathrm{Cl})=0.9327$ ). Isolates are labelled as described and are denoted by respective isolate (MSHR) ID's. Red stars on branches denote bootstrap values $<80 \%$

bed isolates nor the ST-36 air sample isolate matching any of the eight meerkat genomes. Despite this, our data collectively showed that most of the cases were likely related to a single strain, with gross and histopathological examination suggesting two main routes of infection: $\mathrm{cu}-$ taneous inoculation and ingestion.

Data from the ST-36 reconstructed phylogeny revealed only minor differences across the seven meerkat strains, with infecting genomes clustering closely together on one branch of the tree. While the ST-36 air sample and black-capped capuchin isolates were also collected from the same Wildlife Park, they did not group together with the meerkat isolates, further indicating that the seven meerkat B. pseudomallei strains were epidemiologically related to one another. The $B$. pseudomallei genome has an exceedingly high rate of lateral gene transfer and recombination [29] in its natural soil environment. $B$. pseudomallei can also undergo genetic change due to host selective pressures and these changes can range from small genetic changes caused by mutations to large reductive evolutionary changes $[37,38]$. Since the meerkat ST-36 outbreak took place over more than seven-months and samples were isolated at variable time points throughout this period, it could explain why we observed some SNP diversity between isolates. Small SNP variations have been seen in other investigations linking cases of melioidosis to epidemiologically-related environmental B. pseudomallei strains previously [23, 33, 39]. Moreover, all isolates were found to contain the $\operatorname{bim}_{B p}$ rather than the $\operatorname{bim}_{B m}$, locus variant that is normally associated with more severe neurological manifestations of the disease. The B. pseudomallei meerkat isolates were also negative for fhaB3, which has been associated with decreased pathogenicity in previous human studies [7]. This may in part explain the relatively slow disease progression observed in the meerkats, in comparison to the rapid sepsis and death seen in other descriptions of melioidosis in imported exotic animals $[14,40]$.

While B. pseudomallei was not isolated from any sand, soil or swab samples collected from within the enclosure, inoculation through the skin was determined as the 
most likely mechanism of pathogenesis in the majority of cases. Sites of cutaneous infection tended to be centred on sites of previous trauma, such as bony protuberances of the distal limbs, scrotum and tail, suggesting inoculation at sites of skin damage. The deep suppurative skin lesions were the only melioidosis lesions that were associated with fibroplasia, suggesting they were of the longest duration [41], and corroborating that skin inoculation was likely the primary route of infection. As $B$. pseudomallei has been detected in faecal samples of other species including wallabies and chickens [11], it is possible the bacterium was shed into the enclosure by previously infected meerkats and wounds became infected while burrowing or digging around contaminated sand. Likewise, animal-to-animal transmission of the infection may have occurred through lesions and bites sustained from intraspecific aggression among the meerkats. Of note, in an effort to prevent future fighting and decrease stress amongst the remaining meerkats, only males were reintroduced to the enclosure after October 2016, when the final case fatality occurred. Since then no melioidosis infections have been reported in the park meerkats. While animal-to-animal transmission is considered rare, it has been described before in Australian goats and pigs and is believed to occur through wound or nasal secretions, milk, faeces and urine $[9,42]$. Accordingly, transmission through wounds inflicted during fighting likely played some role in infection, which is supported by B. pseudomallei never having been recovered inside the enclosure.

The second main route of infection suggested by the pathology seen in these cases is ingestion, given the severity and relatively long duration (associated fibroplasia) of lesions specifically involving the hepatic portal vessels that drain pathogens entering from the gastrointestinal tract [43]. Meerkats may have a particular susceptibility to the ingestion route of infection given its apparent relative rarity in some other species [3], although in one other reported outbreak of melioidosis in meerkats portal vascular targeting of lesions was not described [21]. It is possible that the presence of skin lesions harbouring B. pseudomallei and infection by ingestion are related, given the grooming habits of meerkats and observation by keepers of the meerkats licking and chewing at skin lesions. Moreover, all food, drinking water and bowl swab samples were culture-negative for the bacterium, though this may have been the result of under-sampling, decontamination of the water bowl, or rotation of the food supply prior to sample collection. Additionally, while detection of the ST-36 air isolate nearby to the enclosure provides support for aerosolised B. pseudomallei being passed into the open-air enclosure and potentially contaminating the drinking water or food supply, the air ST-36 was genetically too distant to the meerkat ST36 isolates to be the infecting strain and this mode of contamination of the enclosure therefore remains only speculative.
Lung lesions in all cases were either random multifocal and/or centred on pulmonary vessels. This is suggestive of bacteraemic embolic spread from the systemic circulation [44] rather than bronchopneumonia from inhalation, which is a common pathogenesis in other species $[3,13,14,45,46]$. The occurrence of most of the cases in the drier months (between April and October) rather than the monsoon season also fits with inhalation not being a major source of infection, since inhalational melioidosis has been shown to occur more frequently during severe-weather related events and heavy monsoonal rains $[3,9]$. In most of the meerkats there were also random multifocal suppurative hepatic foci, suggesting embolic spread from systemic circulation. These random multifocal lesions in the liver and lung, along with culture of $B$. pseudomallei from the spleen, indicate terminal septicaemia due to $B$. pseudomallei occurred in all the meerkats, a common sequela to infection among many species $[6,10,46]$. There was no pathological evidence of chronicity or latency, such as fibrous nodules within organs in any of the meerkats.

\section{Conclusions}

In the current investigation, we used high-resolution WGS concurrently with detailed pathology data to investigate a cluster of fatal B. pseudomallei infections in slender-tailed meerkats. While more traditional B. pseudomallei molecular fingerprinting schemes can often confound inferences about infection aetiology and transmission due to the exceedingly high rate of genetic recombination, our findings demonstrate the epidemiological insights that can be gained from high-throughput WGS. Additionally, our data verifies the importance of WGS for expanding the current knowledge of $B$. pseudomallei genotype diversity and pointsource attribution in animal cases of melioidosis. These veterinary pathology and genomics findings have important "One Health" implications in providing insights into human melioidosis epidemiology regarding both routes of infection and point-source attribution.

While no environmental point-source was determined, seven of the meerkat strains were highly similar on the whole-genome level, implying that the cases were related. Despite this, pathology data indicated that even amongst the seven clonal ST-36 strains, routes of infection likely varied between the meerkats. Collectively, these findings suggest that meerkats are very susceptible to melioidosis and that stress and intraspecific competition amongst the animals can play an important role in infection. Strategies to reduce the risk of infection are imperative for highly susceptible non-native species imported into melioidosis endemic areas, particularly for iconic exotic species on display in zoos and wildlife parks. 


\section{Methods}

\section{Animals and animal sample processing}

The eight deceased slender-tailed meerkats (Suricata suricatta) were from a Wildlife Park in the Darwin region $\left(12.5^{\circ} \mathrm{S}\right)$ of Northern Australia. One female and one male meerkat imported from a Tasmanian zoo in Australia were introduced to the park in late 2014. The index case was the adult male, found dead inside the enclosure in March, 2015. Two additional males were introduced to the park shortly after and two litters totalling eight meerkats were born in mid-2015. Seven subsequent fatal cases of melioidosis occurred in the female matriarch and six of the adolescent meerkats between February and October 2016. For cases \#2 and \#8, the meerkats were observed unwell for 1-2 days prior to being found dead. Otherwise, clinical signs were observed for 1-2 weeks prior to death, and commonly included lethargy, and in some cases, cutaneous sores, which the meerkats were observed chewing or licking at (cases \#3, \#4, \#5, \#8), and limping or hind limb paresis (cases \#3, \#7, \#8). Several meerkats that were observed to be unwell were treated with $10 \mathrm{mg} / \mathrm{kg}$ doxycycline administered orally in food treats once daily (Psittavet, Vetafarm, Wagga Wagga, NSW). Two cases were treated with cefovexin, $8 \mathrm{mg} / \mathrm{kg}$ injected subcutaneously (Convenia, Zoetis, Rhodes, NSW); case \#2 was treated twice, once at five weeks and again four days prior to death, and case \#3 was treated one day prior to death. Treated animals were allowed to remain with the colony to minimise stress.

Complete gross post-mortem examinations were performed on the eight deceased meerkats at Berrimah Veterinary Laboratories (BVL). For histology, tissue samples were fixed in $10 \%$ phosphate buffered formalin, processed in standard fashion and stained with haematoxylin and eosin. A wide range of tissues were examined histologically for each meerkat, including liver, lung, spleen, heart, kidney and brain, plus stomach and multiple sections of intestine in the better-preserved carcasses, and bones or skin in selected carcasses in which the clinical history suggested possible involvement or where gross lesions were observed.

Post-mortem samples for bacterial culture were collected aseptically and selected case by case based on whether the tissue exhibited a gross lesion or could add to information on pathogenesis (Table 1). For aerobic bacterial culture swabs of lesions or tissue samples were homogenised with physiological saline, placed in Ashdown broth and incubated at $35^{\circ} \mathrm{C}$ for seven days for enrichment culture. Samples were then plated onto Tryptic soy agar with sheep's blood, MacConkey and Ashdown agars (Thermo Fisher Scientific, Thebarton, Australia) and incubated at $35^{\circ} \mathrm{C}$ for $48 \mathrm{~h}$ (Additional file 1: Fig. S1). Isolates were identified using the API 20
NE system (bioMérieux, Marcy l'Etoile, France). Those classified as B. pseudomallei were transferred to Menzies School of Health Research (Menzies) for further confirmation and strain designation.

In addition to post-mortem samples, samples were taken under general anaesthetic from two meerkats that were in the colony and survived the outbreak to determine if they might be subclinical carriers of B. pseudomallei. Samples included gastric aspirates, throat and rectal swabs. All were placed directly into Ashdown broth and blood was also placed directly into thioglycollate broth.

\section{Environmental sample collection}

Six rounds of environmental sampling were carried out at the park between May 2016 and March 2017 in relation to the meerkat fatalities to ensure thorough sampling of the enclosure and all adjacent park areas. Samples were collected from within the meerkat enclosure, nearby animal enclosures and adjacent garden areas. A total of 64 samples were collected from the park, including 33 soils, five waters, nine environmental swabs, five plants, two food, three faecal samples, and seven air samples collected using a portable high-volume air sampler with gelatin membrane filters (Sartorius MD8, Goettingen, Germany) (Additional file 2 Data set S1).

\section{Environmental sample processing and confirmation}

Culture of B. pseudomallei from water, soil, air and swab specimens was carried out using methods previously developed in our laboratory [23, 27, 47]. Briefly, samples were enriched in Ashdown's broth containing colistin $(50 \mathrm{mg} / \mathrm{L})$ and incubated at $37^{\circ} \mathrm{C}$ aerobically for 2 and 7 days. Enriched broth was plated onto Ashdown's agar with gentamicin $(8 \mathrm{mg} / \mathrm{L})$ and incubated for $48 \mathrm{~h}$, and all colonies resembling $B$. pseudomallei were sub-cultured onto Ashdown's agar. DNA from suspected colonies was extracted using 10\% Chelex-100 resin [48] and confirmation of $B$. pseudomallei was carried out using a realtime PCR assay targeting a $115 \mathrm{bp}$ segment within the type three secretion system 1 (TTS1) gene cluster [49] specific to B. pseudomallei.

\section{Isolates used for comparative phylogenomic analysis}

B. pseudomallei genomes from 13 ST-36 isolates originating from Darwin, Australia were used in the analysis (Additional file 3: Data set S2). Isolates were collected between 1994 and 2017. Eight animal isolates were used in the reconstructed phylogeny: the seven ST-36 meerkat isolates from the outbreak (MSHR9253, MSHR9256, MSHR9460, MSHR9464, MSHR9644, MSHR9647, MSHR9650); and one isolate from a black-capped capuchin (Sapajus apella) (MSHR7950). The black-capped capuchin was an exotic import on display at the same Wildlife Park and died from melioidosis in 2012. Also 
included in the ST-36 phylogenetic analysis were one air sample (MSHR10004) collected as part of the park environmental survey and four isolates from human infections collected as part of the Darwin Prospective Melioidosis Study (Human Research Ethics Committee of the Northern Territory Department of Health and the Menzies School of Health Research, approval HREC 02-38) [3] (MSHR0305, MSHR2078, MSHR9698, MSHR10679) (Additional file 3: Data set S2).

\section{Whole-genome sequencing of clinical and environmental Wildlife Park isolates}

Genomic DNA was extracted using the Qiagen DNeasy blood and tissue kit (Qiagen, Chadstone, Victoria, Australia) as previously described [24]. Isolates were sequenced at Macrogen, Inc. (Gasan-dong, Seoul, Republic of Korea) or Australian Genome Research Facility Ltd. (Melbourne, Australia) using the Illumina HiSeq2000 and Illumina HiSeq2500 platforms (Illumina, Inc., San Diego, CA).

Identification of orthologous core genome singlenucleotide polymorphism (SNP) and small insertion or deletion (InDel) variants from WGS data was performed using SPANDx [50]. MSHR0305, an isolate obtained from a Darwin melioidosis patient in 1994, was used as a high-quality ST-36 reference assembly (N50: 4054155, total length: 7428072 , contigs: $n=2$ ). Phylogenetic reconstruction was based on maximum-parsimony in PAUP v4.0a162 [51] using both SNP and InDel variants to increase resolution [33]. Bootstrapping with 1000 replicates was carried out to determine robustness of branches. Phylogenetic trees were examined in FigTree v1.4.4 (http://tree.bio.ed.ac.uk/software/figtree/) and managed using the online tool iTOL v3: Interactive Tree of Life [52].

\section{Multi-locus sequence typing (MLST) assignment}

MLST types for human clinical isolates $(n=4)$ were assigned from WGS data in silico prior to the study commencing using the BIGSdb tool accessible on the $B$. pseudomallei MLST website (http://pubmlst.org/bpseudomallei/) [28, 53]. MLST assignment of B. pseudomallei meerkat isolates $(n=8)$, black-capped capuchin isolate $(n=1)$ and environmental isolates collected as part of the park survey $(n=3)$ was done utilising a series of inhouse PCR primers targeting the common Darwin ST types 36, 109, 132 and 562 [31]. ST types were later confirmed using WGS data assigned in silico.

\section{Virulence factor assignment}

Virulence factor fhaB3 and $\operatorname{bim}_{B m / B p}$ positivity were determined using the online basic local alignment search tool (BLAST) as previously described [54]. In brief, the genes were BLAST searched against the B. pseudomallei
WGS using the nucleotide BLAST (BLASTn) parameter, and each genome was assigned as fhaB3 (encoded by BPSS2053 in B. pseudomallei K96243) positive or negative, and as a carrier of $B i m A_{B m}$ (BURPS668_A2118 in B. pseudomallei MSHR668) or $B i m A_{B p}$ (BPSS1492 in $B$. pseudomallei K96243).

\section{Supplementary information}

Supplementary information accompanies this paper at https://doi.org/10. 1186/s12917-019-2198-9.

Additional file 1: Figure S1. Clinical microbiological detection of $B$. pseudomallei in meerkats B. pseudomallei growth on Sheep's Blood Agar (A.), and growth on selective Ashdown Agar (B.) Gram-negative bacilli characteristic of B. pseudomallei as seen by Gram-stain (C.) (bar=10 $\mu \mathrm{m})$

Additional file 2: Data set S1. Data set 1- Environmental samples collected at the Wildlife Park in response to the meerkat case cluster

Additional file 3 Data set S2. Data set 2- ST-36 isolates used for WGS phylogenetic comparison

\section{Abbreviations}

B. pseudomallei: Burkholderia pseudomallei; BVL: Berrimah Veterinary Laboratories; InDel: Insertion and/or deletion; MLST: Multi-locus sequence type; NT: Northern Territory; SNP: Single nucleotide polymorphism; ST: Sequence type; WGS: Whole-genome sequencing

\section{Acknowledgements}

We thank the staff of Crocodylus Park, NT and Prof. Graham J. G. Webb and Dr. Charlie Manolis for their expert advice and allowing us access to the Wildlife Park. We also thank Glenda Harrington and Barbara Machunter for their laboratory support, as well as microbiology staff at the Royal Darwin Hospital and Berrimah Veterinary Laboratory for supplying B. pseudomallei human and animal isolates. Kitman Dyrting at Berrimah Veterinary Laboratories performed one of the gross post-mortems.

\section{Ethical approval and consent to participate}

Approval for patient data was attained through the Human Research Ethics Committee of the Northern Territory Department of Health and Families (HREC 02/38) and Menzies. Patient data was made anonymous prior to the study commencing. Animal ethics approval was not required as meerkats were under normal zoological settings, clinical veterinary care and died of natural causes (they were not experimental subjects). However, approval for work with animal samples was obtained by Menzies School of Health Research and Berrimah Veterinary Laboratory before commencement of the study. Post-mortems were performed at Berrimah Veterinary Laboratory on a routine diagnostic basis. Consent to participate in the study and publication of results was obtained by Crocodylus Park and Berrimah Veterinary Laboratories prior to collection and use of deceased meerkat samples.

\section{Authors' contributions}

$A R$, VR, MM, and BJC oversaw environmental data collection and sample processing. CS, SB and IG oversaw clinical pathology and microbiology sample processing from meerkats. AR, MM, BJC and MK conceived the study analysis. AR, MK, JW, and MK did the analyses. AR, MM, MK, CS and BJC contributed to the writing of the manuscript. All study authors reviewed and approved the final manuscript.

\section{Funding}

The research presented was funded under the Australian National Health and Medical Research Council: grant numbers 1046812, 1098337 and 1131932 (The HOT NORTH initiative). AR is funded by a Charles Darwin International PhD scholarship. The funding bodies played no role in study design, analysis or writing of the manuscript.

\section{Availability of data and materials}

The dataset supporting the conclusions of this article is available in the National Center for Biotechnology Information Short Read Archive, under 
BioProject accession number PRJNA532306 (https://www.ncbi.nlm.nih.gov/ bioproject/532306), accessions SAMN11394028-SAMN11394038.

\section{Consent for publication}

Not applicable.

\section{Competing interests}

The authors declare that there are no competing interests.

\section{Author details}

${ }^{1}$ Menzies School of Health Research, Charles Darwin University, Darwin, Casuarina NT 0811, Australia. ${ }^{2}$ Department of Primary Industry and Resources, Berrimah Veterinary Laboratory, Berrimah Farm, Makagon Road, Berrimah, Northern Territory 0828, Australia. ${ }^{3}$ Research Institute for the Environment and Livelihoods, Charles Darwin University, Darwin, Northern Territory 0811 Australia. ${ }^{4}$ Parap Veterinary Hospital, Parap, Darwin, Northern Territory 0820, Australia. ${ }^{5}$ Royal Darwin Hospital and Northern Territory Medical Program, Darwin, Northern Territory 0811, Australia.

\section{Received: 23 May 2019 Accepted: 27 November 2019}

\section{Published online: 19 December 2019}

\section{References}

1. Wiersinga WJ, Currie BJ, Peacock SJ. Melioidosis. N Engl J Med. 2012:367(11): 1035-44.

2. Cheng AC, Currie BJ. Melioidosis: epidemiology, pathophysiology, and management. Clin Microbiol Rev. 2005;18(2):383-416.

3. Currie BJ, Ward L, Cheng AC. The epidemiology and clinical spectrum of melioidosis: 540 cases from the 20 year Darwin prospective study. PLoS Negl Trop Dis. 2010;4(11):e900.

4. Currie BJ, Mayo M, Anstey NM, Donohoe P, Haase A, Kemp DJ. A cluster of melioidosis cases from an endemic region is clonal and is linked to the water supply using molecular typing of Burkholderia pseudomallei isolates. Am J Trop Med Hyg. 2001;65(3):177-9.

5. Limmathurotsakul D, Kanoksil M, Wuthiekanun V, Kitphati R, deStavola B, Day NP, Peacock SJ. Activities of daily living associated with acquisition of melioidosis in Northeast Thailand: a matched case-control study. PLoS Negl Trop Dis. 2013;7(2):e2072.

6. Wiersinga WJ, van der Poll T, White NJ, Day NP, Peacock SJ. Melioidosis: insights into the pathogenicity of Burkholderia pseudomallei. Nat Rev Microbiol. 2006;4(4):272-82.

7. Sarovich DS, Price EP, Webb JR, Ward LM, Voutsinos MY, Tuanyok A, Mayo $M$, Kaestli M, Currie BJ. Variable virulence factors in Burkholderia pseudomallei (melioidosis) associated with human disease. PLoS One. 2014; 9(3):e91682.

8. Low Choy J, Mayo M, Janmaat A, Currie BJ. Animal melioidosis in Australia. Acta Trop. 2000;74(2-3):153-8.

9. Currie BJ. Melioidosis: evolving concepts in epidemiology, pathogenesis, and treatment. Semin Respir Crit Care Med. 2015;36(1):111-25.

10. Spraque LD, Neubauer H. Melioidosis in animals: a review on epizootiology, diagnosis and clinical presentation. J Vet Med B Infect Dis Vet Public Health. 2004:51(7):305-20.

11. Hoger AC, Mayo M, Price EP, Theobald V, Harrington G, Machunter B, Choy $\mathrm{J}$, Currie BJ, Kaestli M. The melioidosis agent Burkholderia pseudomallei and related opportunistic pathogens detected in faecal matter of wild life and livestock in northern Australia. Epidemiol Infect. 2016;144(9):1924-32.

12. Limmathurotsakul $D$, Thammasart $S$, Warrasuth $N$, Thapanagulsak $P$, Jatapai A, Pengreungrojanachai V, Anun S, Joraka W, Thongkamkoon P, Saiyen P, et al. Melioidosis in animals, Thailand, 2006-2010. Emerg Infect Dis. 2012; 18(2):325-7.

13. Sim S, Ong C, Gan Y, Wang D, Koh V, Tan Y, Wong M, Chew J, Ling S, Tan B, et al. Melioidosis in Singapore: clinical, veterinary, and environmental perspectives. Trop Med Infect Dis. 2018;3(1):31.

14. Kasantikul T, Sommanustweechai A, Polsrila K, Kongkham W, Chaisongkram C, Sanannu S, Kongmakee P, Narongwanichgarn W, Bush M, Sermswan RW, et al. Retrospective study on fatal Melioidosis in captive zoo animals in Thailand. Transbound Emerg Dis. 2016;63(5):E389-94.

15. Cottew GS. Melioidosis in sheep in Queens land; a description of the causal organism. Aust J of Exp Biol Med Sci. 1950;28(6):677-83.

16. Forbes-Faulkner JC, Townsend WL, Thomas AD. Pseudomonas pseudomallei infection in camels. Aust Vet J. 1992;69(6):148.
17. Janmaat A, Low Choy J, Currie B. Melioidosis in an alpaca (Lama pacos). Aust Vet J. 2004;82(10):622-33.

18. Tonpitak W, Sornklien C, Chawanit M, Pavasutthipaisit S, Wuthiekanun V, Hantrakun V, Amornchai P, Thaipadungpanit J, Day NPJ, Yingst S, et al. Short report: fatal Melioidosis in goats in Bangkok, Thailand. Am J Trop Med Hyg. 2014;91(2):287-90.

19. Ketterer P, Webster W, Shield J, Arthur R, Blackall P, Thomas A. Melioidosis in intensive piggeries in south eastern Queensland. Aust Vet J. 1986;63(5):146-9.

20. Dance D, King C, Aucken H, Knott C, West P, Pitt T. An outbreak of melioidosis in imported primates in Britain. Vet Rec. 1992;130(24):525-9.

21. Kongmakee UM, Noimoon S, Banlunara W. Melioidosis in Captive Slender Tailed Meerkats (Suricata suricatta). In: Proceedings of the 14th Chulalongkorn University Veterinary Conference; 2015 Apr 20-22; Bangkok, Thailand: p. 211-212.

22. Sarovich DS, Garin B, De Smet B, Kaestli M, Mayo M, Vandamme P, Jacobs J, Lompo P, Tahita MC, Tinto H, et al. Phylogenomic Analysis Reveals an Asian Origin for African Burkholderia pseudomallei and Further Supports Melioidosis Endemicity in Africa. mSphere. 2016;1 (2).

23. Currie BJ, Price EP, Mayo M, Kaestli M, Theobald V, Harrington I, Harrington G, Sarovich DS. Use of whole-genome sequencing to link Burkholderia pseudomallei from air sampling to Mediastinal Melioidosis, Australia. Emerg Infect Dis. 2015;21(11):2052-4.

24. Currie BJ, Gal D, Mayo M, Ward L, Godoy D, Spratt BG, LiPuma JJ. Using BOXPCR to exclude a clonal outbreak of melioidosis. BMC Infect Dis. 2007;7:68.

25. Chapple SNJ, Price EP, Sarovich DS, McRobb E, Mayo M, Kaestli M, Spratt BG, Currie BJ. Burkholderia pseudomallei genotype distribution in the Northern Territory, Australia. Am J Trop Med Hyg. 2016;94(1):68-72.

26. Godoy D, Randle G, Simpson AJ, Aanensen DM, Pitt TL, Kinoshita R, Spratt BG. Multilocus sequence typing and evolutionary relationships among the causative agents of Melioidosis and Glanders, Burkholderia pseudomallei and Burkholderia mallei. J Clin Microbiol. 2003;41(5):2068-79.

27. Mayo M, Kaesti M, Harrington G, Cheng AC, Ward L, Karp D, Jolly P, Godoy D, Spratt BG, Currie BJ. Burkholderia pseudomallei in unchlorinated domestic bore water, Tropical Northern Australia. Emerg Infect Dis. 2011;17(7):1283-5.

28. Price EP, MacHunter B, Spratt BG, Wagner DM, Currie BJ, Sarovich DS. Improved multilocus sequence typing of Burkholderia pseudomallei and closely related species. J Medical Microbiol. 2016;65(9):992-7.

29. Pearson T, Giffard P, Beckstrom-Sternberg S, Auerbach R, Hornstra $H$, Tuanyok A, Price EP, Glass MB, Leadem B, Beckstrom-Sternberg JS, et al. Phylogeographic reconstruction of a bacterial species with high levels of lateral gene transfer. BMC Biol. 2009;7:78.

30. Dale J, Price EP, Hornstra H, Busch JD, Mayo M, Godoy D, Wuthiekanun V, Baker A, Foster JT, Wagner DM, et al. Epidemiological tracking and population assignment of the non-clonal bacterium, Burkholderia pseudomallei. PLoS Negl Trop Dis. 2011;5(12):e1381.

31. Price EP, Sarovich DS, Smith EJ, MacHunter B, Harrington G, Theobald V, Hall CM, Hornstra HM, McRobb E, Podin Y, et al. Unprecedented Melioidosis cases in northern Australia caused by an Asian Burkholderia pseudomallei strain identified by using large-scale comparative genomics. Appl Environ Microbiol. 2016;82(3):954-63.

32. Aziz A, Sarovich DS, Harris TM, Kaestli M, McRobb E, Mayo M, Currie BJ, Price EP. Suspected cases of intracontinental Burkholderia pseudomallei sequence type homoplasy resolved using whole-genome sequencing. Microb Genom. 2017;3(11):e000139.

33. McRobb E, Sarovich DS, Price EP, Kaestli M, Mayo M, Keim P, Currie B. Tracing Melioidosis Back to the source: using whole-genome sequencing to investigate an outbreak originating from a contaminated domestic water supply. J Clin Microbiol. 2015;53(4):1144.

34. Stevens MP, Stevens JM, Jeng RL, Taylor LA, Wood MW, Hawes P, Monaghan P, Welch MD, Galyov EE. Identification of a bacterial factor required for actin-based motility of Burkholderia pseudomallei. Mol Microbiol. 2005;56(1):40-53.

35. Dodin A, Galimand M. origin, course and recession of an infectious disease, melioidosis, in temperate countries. Arch Inst Pasteur Tunis. 1986;63(1):69-73.

36. Ralph A, McBride J, Currie BJ. Transmission of Burkholderia pseudomallei via breast milk in northern Australia. Pediatr Infect Dis J. 2004;23(12):1169-71.

37. Limmathurotsakul D, Holden MTG, Coupland P, Price EP, Chantratita N, Wuthiekanun V, Amornchai P, Parkhill J, Peacock SJ. Microevolution of Burkholderia pseudomallei during an acute infection. J Clin Microbiol. 2014; 52(9):3418. 
38. Price E, Hornstra HM, Limmathurotsakul D, Max TL, Sarovich D, Vogler AJ, Dale JL, Ginther JL, Leadem B, Colman RE, et al. Within-host evolution of Burkholderia pseudomallei in four cases of acute melioidosis. PLoS Path. 2010;6(1):e1000725.

39. Sarovich DS, Chapple SNJ, Price EP, Mayo M, Holden MTG, Peacock SJ, Currie BJ. Whole-genome sequencing to investigate a non-clonal melioidosis cluster on a remote Australian island. Microb Genom. 2017;3(8): e000117.

40. Hicks C, Kinoshita R, Ladds P. Pathology of melioidosis in captive marine mammals. Aust Vet J. 2000;78(3):193-5.

41. Zachary JF, McGavin MD. In: Pathologic basis of veterinary disease-E-book: 5th edition. St. Louis: Elsevier Health Sciences; 2013. p.120-127.

42. Rogers RJ, Andersen DJ. Intra-uterine infection of a pig by Pseudomonas pseudomallei. Aust Vet J. 1970;46(6):292.

43. Cullen J, Brown DL. Hepatobiliary system and exocrine pancreas. Pathol Basis Vet Dis. 2012;5:405-57.

44. Lopez A, Martinson SA. Respiratory system, mediastinum and pleurae. Pathol Basis Vet Dis. 2017:471-560.

45. Omar A. Pathology of melioidosis in pigs, goats and a horse. J Comp Pathol Ther. 1963;73:359-IN334.

46. Ketheesan N. Melioidosis: a century of observation and research. Amsterdam: Elsevier; 2012.

47. Limmathurotsakul D, Dance DAB, Wuthiekanun V, Kaestli M, Mayo M, Warner J, Wagner DM, Tuanyok A, Wertheim H, Yoke Cheng T, et al. Systematic review and consensus guidelines for environmental sampling of Burkholderia pseudomallei. PLoS Negl Trop Dis. 2013;7(3):e2105.

48. de Lamballerie X, Zandotti C, Vignoli C, Bollet C, de Micco P. A one-step microbial DNA extraction method using "Chelex 100" suitable for gene amplification. Res Microbiol. 1992;143(8):785-90.

49. Novak RT, Glass MB, Gee JE, Gal D, Mayo MJ, Currie BJ, Wilkins PP. Development and evaluation of a real-time PCR assay targeting the type III secretion system of Burkholderia pseudomallei. J Clin Microbiol. 2006;44(1): 85-90.

50. Sarovich DS, Price EP. SPANDx: a genomics pipeline for comparative analysis of large haploid whole genome re-sequencing datasets. BMC Res. 2014;7: 618.

51. Wilgenbusch JC, Swofford D: Inferring evolutionary trees with PAUP*. Curr Protoc Bioinformatics 2003, Chapter 6:Unit 64.

52. Letunic I, Bork P. Interactive tree of life (iTOL) v3: an online tool for the display and annotation of phylogenetic and other trees. Nucleic Acids Res. 2016;44(W1):W242-5.

53. Jolley KA, Maiden MCJ. BIGSdb: scalable analysis of bacterial genome variation at the population level. BMC Bioinform. 2010;11:595.

54. Webb JR, Sarovich DS, Price E, Ward LM, Mayo M, Currie BJ. Burkholderia pseudomallei lipopolysaccharide genotype does not correlate with severity or outcome in melioidosis: host risk factors remain the critical determinant. Open Forum Infect Dis. 2019;6(4):ofz091.

\section{Publisher's Note}

Springer Nature remains neutral with regard to jurisdictional claims in published maps and institutional affiliations.

Ready to submit your research? Choose BMC and benefit from:

- fast, convenient online submission

- thorough peer review by experienced researchers in your field

- rapid publication on acceptance

- support for research data, including large and complex data types

- gold Open Access which fosters wider collaboration and increased citations

- maximum visibility for your research: over $100 \mathrm{M}$ website views per year

At BMC, research is always in progress.

Learn more biomedcentral.com/submissions 